\title{
Relay-Assisted MIMO Transmission with Three-Phase Scheduling in Cognitive Radio Networks
}

\author{
Sen Shao*, Qingmin Meng*†, Wenhui Zong* and Wei-ping Zhu*‡ \\ ${ }^{*}$ Institute of Signal and Transmission \\ Nanjing University of Posts and Telecommunications, Nanjing, 210003, China \\ ${ }^{\dagger}$ National Mobile Communications Research Lab, Southeast University, Nanjing 210096, China \\ ${ }_{\ddagger}^{\ddagger}$ Centre for Signal Processing and Communications \\ Dept. of Electrical and Computer Engineering, Concordia University, Montreal, Quebec, Canada H3G 1M8
}

\begin{abstract}
In this paper, we investigate a joint scheduling and transmitting scheme in cognitive radio networks. Three phases are considered in the proposed scheme(named scheme 1): 1)in the first phase, $N_{t}$ primary users(PUs) in the high-rate region are chosen to implement communication with primary base station (PBS); 2)in the second phase, PBS will support simultaneously the fixed relay station(RS) and the selected PUs in the highrate region based on certain beamforming $(\mathrm{BF}) ; 3)$ in the third phase, selected PUs in the low-rate region and selected cognitive users(CUs) in the cognitive region are supported by RS. In particular, during the phases above, all selected users send back their channel covariance matrices to PBS or RS on the purpose of lowering the feedback and implementing certain BF. Furthermore, a frame structure used for the joint scheduling and transmitting scheme is presented and the performance of scheme 1 with imperfect channel covariance matrix feedback is evaluated to compare with that of scheme 1 with perfect feedback. From the simulation results, the proposed scheme(scheme 1) can significantly improve the throughput of the network over the basic system(named scheme 2) without spectral sharing.
\end{abstract}

\section{INTRODUCTION}

According to the measurements of Federal Communication Commission (FCC) recently, roughly 90 percent licensed frequency bands remain idle[1]. However, intense competition has arisen, especially for the spectrum below $3 \mathrm{GHz}$ owing to the tremendous enhancement of wireless communication. Accordingly, the availability of the licensed wireless spectrum becomes extraordinarily limited. The low spectrum usage should be raised. From the point of view of J.Mitola[2], cognitive radio(CR) can be used for enhancing the spectral usage efficiency by permitting one cognitive user(CU) or more to share the spectrum resources with one licensed user(primary user, PU) or more. By sensing the spectral holes, CUs are capable of accessing the licensed spectrum without interfering PUs. As a result, spectral usage efficiency increases. Referring to the recent work by A.Goldsmith[3], CR technology includes three main cognitive radio paradigms: underlay, overlay, interweave. Our proposed system of scheme 1 based on the combination of the underlay and overlay pattern will be mainly discussed in this paper.

Relay transmission strategy in the cellular system was proposed to cover dead spots, such as subways, tunnels or inbuilding areas, where a base station(BS) is not able to support the mobile users[4]. The basic idea of relay transmission is to deploy intermediate nodes which can forward the received data from the source to the destination. Furthermore, the relayassisted system has certain potential advantages including the improvement of system throughput and the enhancement of system performance[5] by enlarging the coverage area of a BS. In this work, to maintain the quality of service(QoS) of the primary users far away from PBS(i.e., PUs in the lowrate region), a cognitive base station(CBS) is deployed as a relay station(RS) to support such PUs while carrying out communication with certain CUs.

$\mathrm{BF}$ is a critical technology for multiuser systems. The concept $\mathrm{BF}$ derives from the requirement of receiving a signal radiating from a specific transmitter and attenuating signals from other transmitters. Zero-forcing beamforming(ZFBF)[6] is a suboptimal but practical strategy where the beamforming weight matrix is computed to avoid interference among user streams. However, ZFBF has some drawbacks that it is only known to work for one receive antenna per user[7]. Recent work on cognitive multiuser multiple input/multiple output(MIMO) systems[8] has adopted antenna selection to guarantee one receive antenna per user, which makes it possible to compute ZFBF weighting vectors at the transmitter. In this paper, similar to [9], beamforming weight vectors are chosen based on the corresponding optimization problem using the properties of generalized eigenvalue decomposotion. Such beamforming vectors are capable of reducing the inter-user interference among the selected users.

The rest of the paper is organized as follows. In section II, the system models of our proposed schemes are presented. Then in section III the beamforming approach and the frame structure design will be discussed in detail. Performance analysis and simulation results will be given in section IV and in section V. Finally, we will come to a conclusion in section VI. Throughout this paper, we use uppercase boldface letters for matrices and lowercase boldface letters for vectors. The Frobenius norm of vectors are denoted by $\|.\|_{\mathrm{F}} ;(.)^{H}$ 


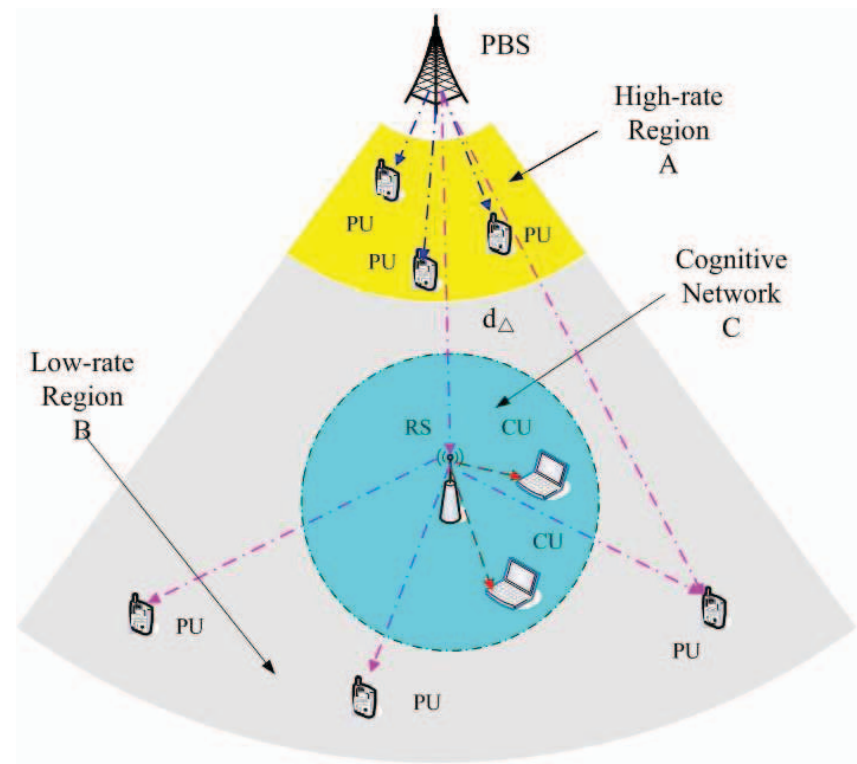

Fig. 1. System model of scheme 1

and $\mathrm{E}($.$) stands for the conjugate transpose operator and the$ expectation operator respectively.

\section{System Model}

\section{A. System Models of Scheme 1 and Scheme 2}

The system of scheme 1 is a multiuser MIMO system with the coexistence of a CR network that shares the same spectrum for transmission with an existing primary celluar network. The downlink consisting of $\mathrm{PBS} \rightarrow P U_{N}$ link, $\mathrm{PBS} \rightarrow \mathrm{RS}$ link, $\mathrm{RS} \rightarrow \mathrm{CU}$ link and $\mathrm{RS} \rightarrow P U_{F}$ link is considered. $P U_{N}$ and $P U_{F}$ denotes the PUs in the high-rate region(A area) and low-rate region(B area) respectively. The deployment scenario of our proposed system is shown in Fig.1. A single isolate $60^{\circ}$ sector with a coverage radius $600 \mathrm{~m}$ is considered. RS lies at the center of the sector. PBS and RS are located above rooftops with direct links and have arrays with $N_{t}$ antenna elements and both PUs and CUs are equipped with $N_{r}$ omnidirectional antennas. The flat Rayleigh fading channel model between PBS and PU, RS and PU, RS and CU, and Ricean fading channel model between PBS and RS are supposed. $K_{A}, K_{B}$ respectively denotes the number of PUs uniformly distributed in $\mathrm{A}$ and $\mathrm{B}$ area, while $K_{C}$ denotes the number of CUs uniformly distributed in $\mathrm{C}$ area. RS amplifies and forwards the symbols received from PBS to the selected PUs in $\mathrm{B}$ area.

The system of scheme 2 is similar to that of scheme 1 except that there are no CUs and RS. So only the downlink consisting of $\mathrm{PBS} \rightarrow P U_{N}$ link and $\mathrm{PBS} \rightarrow P U_{F}$ link is considered. Scheme 2 is a common celluar network which is used as the comparison of the performance with scheme 1 .
Frame of scheme 1

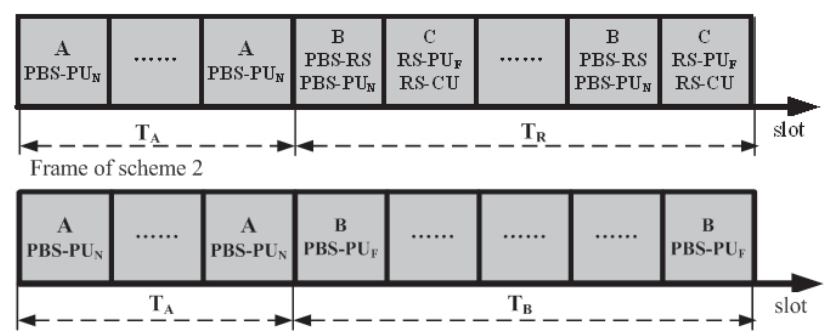

Fig. 2. Frame structure for the two schemes

\section{B. Operating Mechanism of the CR Network and the Primary Cellular Network in Scheme 1}

Both the system of scheme 1 and scheme 2 apply the TDMA strategy in the MIMO downlinks. According to the frame structure discussed in section III, PBS selects $N_{t}$ PUs in A area to communicate in $\mathrm{A}$ time slots while suspending the transmission of the PUs in B area and CUs in C area; in B time slots, RS(acting as the special PU) and ( $\left.N_{t}-1\right)$ PUs in A area are allowed to receive the data from PBS while keeping PUs in B area and CUs in $\mathrm{C}$ area silent; in $\mathrm{C}$ time slots, RS let PUs in B area and CUs in $\mathrm{C}$ area simultaneously share the spectrum while keeping PBS and PUs in A area from work. In a word, only in $\mathrm{C}$ time slots can $\mathrm{CR}$ network be activated to share the spectrum with the primary cellular network(B area). RS serves two different functions in scheme 1: 1) assisting the transmission of $P U_{F}$ in $\mathrm{B}$ area. 2) acting as a CBS to enable the CUs in $\mathrm{C}$ area to share the same spectrum with the PUs in area B through coordinating with the primary network. In this paper, we assume the transmitter(PBS or RS) don't know the perfect channel state information while each PUs and CUs have perfect channel knowledge and the selected users send back the channel covariance matrix $\mathbf{R}$ to PBS or RS. By using beamforming approach in section III, the transmitter can calculate the beamforming vectors to null the inter-user interferences.

\section{Derivation of Sum-rate}

We present the method of calculating the sum-rate of one time slot, which can be applied to the analysis of A and B time slots in scheme 1 and 2 . The frame structure design and the expression of sum-rate in the $\mathrm{C}$ time slots in scheme 1 will be depicted in detail in section III.

In the studied scheme as shown in Fig.1., we suppose that $N_{t} \ll K_{A}+K_{B}+K_{C}$ is satisfied and both the large scale path loss and small scale fading are considered. $n$ denotes the path loss exponent and we drop the log-normal shadowing effect for simplicity. Assume in each time slot, the transmitter selects $N_{t}$ users to receive data, so a block of information symbols $\mathbf{s}=\left[s_{1}, s_{2}, \cdots, s_{N_{t}}\right]^{T}$ with uncorrelated unit-power entries are transmitted to the selected users. The channel and the distance between the transmitter and user $k$ are represented by a $N_{r} \times N_{t}$ matrix $\mathbf{H}_{k}$ and $d_{k}$. $P_{B S}$ stands for the transmitted power of each user. Let $s_{k}$ denote the transmitted 
symbol to the $k$ user, and $\mathbf{n}_{k}$ be the additive white Gaussian noise vector observed at the receiver whose elements are i.i.d complex Gaussian with zero mean and $\sigma^{2}$ variance. Let $\mathbf{w}_{k}$ and $\mathbf{f}_{k}$ denote the unit-norm transmit beamforming and receive combining vectors. Then with the maximal ratio combining receiver $\left(i . e ., \mathbf{f}=\mathbf{H w} /\|\mathbf{H w}\|_{F}\right)$, the signal at the $k$ user is

$r_{k}=\mathbf{f}_{k}{ }^{H} \mathbf{H}_{k} \mathbf{w}_{k} \sqrt{\frac{P_{B S}}{d_{k}{ }^{n} N_{t}}} s_{k}+\sum_{q \neq k} \mathbf{f}_{k}{ }^{H} \mathbf{H}_{k} \mathbf{w}_{q} \sqrt{\frac{P_{B S}}{d_{k}{ }^{n} N_{t}}} s_{q}+\widetilde{n_{k}}$

where $\widetilde{n_{k}}=\mathbf{f}_{k}{ }^{H} \mathbf{n}_{k}, \mathbf{f}_{k}=\mathbf{H}_{k} \mathbf{w}_{k} /\left\|\mathbf{H}_{k} \mathbf{w}_{k}\right\|_{F}$. The sum-rate in one time slot can be written as

$$
R=\sum_{k=1}^{N_{t}} \log _{2}\left(1+S I N R_{k}\right)
$$

where $S I N R_{k}$ denotes the signal-to-interference and noise ratio of the $k$ user. From equation (1), $S I N R_{k}$ can be expressed as

$$
S I N R_{k}=\frac{P_{B S} / N_{t}\left|G_{k, k}\right|^{2} d_{k}^{-n}}{\sum_{q \neq k} P_{B S} / N_{t}\left|G_{k, q}\right|^{2} d_{k}^{-n}+\sigma^{2}}
$$

where $G_{k, k}=\left\|\mathbf{H}_{k}\right\|_{F}^{2} *\left(\mathbf{w}_{k}^{\mathbf{H}} \mathbf{R}_{k} \mathbf{w}_{k}\right) /\left\|\mathbf{H}_{k} \mathbf{w}_{k}\right\|_{F}, G_{k, q}=$ $\left\|\mathbf{H}_{k}\right\|_{F}^{2} *\left(\mathbf{w}_{k}^{H} \mathbf{R}_{k} \mathbf{w}_{q}\right) /\left\|\mathbf{H}_{k} \mathbf{w}_{k}\right\|_{F}$ and $\mathbf{R}_{k}=\mathbf{H}_{k}^{H} \mathbf{H}_{k} /\left\|\mathbf{H}_{k}\right\|_{F}^{2}$.

\section{BEAMFORMING AND FRAME STRUCTURE}

\section{A. Beamforming Approach Using the Generalized Eigenvalue} Decomposition

The specific configuration $N_{t}=N_{r}=2$ is only considered in our proposed systems of scheme 1 and scheme 2. We extend the beamforming approach introduced in [9] to the downlink cellular system with spectral sharing(scheme 1). This technique exploits the properties of the generalized eigenvalue decomposition to jointly optimize transmit beamforming and receive combining vectors. This beamforming solution works for the system with two or more transmit and receive antennas and where only two selected users can be served during each time slot.

In each $\mathrm{A}, \mathrm{B}, \mathrm{C}$ time slot, the transmitter selects two different users with the indices being $i$ and $j(i \neq j)$ to communicate while keeping others silent. In A time slots, $i$ and $j$ denote the two selected PUs in A area; in B time slots, $i$ and $j$ stand for RS and a selected PU in A area while $i$ and $j$ indicate a selected PU in B area and a selected CU in $\mathrm{C}$ area during $\mathrm{C}$ time slots. The signals received at $i$ and $j$ user after receiver combining are

$$
\begin{gathered}
r_{i}=\mathbf{f}_{i}{ }^{H} \mathbf{H}_{i} \mathbf{w}_{i} \sqrt{\frac{P_{B S}}{d_{i}{ }^{n} N_{t}}} s_{i}+\mathbf{f}_{i}{ }^{H} \mathbf{H}_{i} \mathbf{w}_{j} \sqrt{\frac{P_{B S}}{d_{i}{ }^{n} N_{t}}} s_{j}+\widetilde{n}_{i} \\
r_{j}=\mathbf{f}_{j}{ }^{H} \mathbf{H}_{j} \mathbf{w}_{i} \sqrt{\frac{P_{B S}}{d_{j}{ }^{n} N_{t}}} s_{i}+\mathbf{f}_{j}{ }^{H} \mathbf{H}_{j} \mathbf{w}_{j} \sqrt{\frac{P_{B S}}{d_{j}{ }^{n} N_{t}}} s_{j}+\widetilde{n_{j}}
\end{gathered}
$$

Then on the condition of the zero inter-user interference constraint $\mathbf{f}_{i}{ }^{H} \mathbf{H}_{i} \mathbf{w}_{j}=0, \mathbf{f}_{j}{ }^{H} \mathbf{H}_{j} \mathbf{w}_{i}=0\left(\left|\mathbf{w}_{i}^{H} \mathbf{R}_{i} \mathbf{w}_{j}\right|=\right.$ $\left.0,\left|\mathbf{w}_{j}^{H} \mathbf{R}_{j} \mathbf{w}_{i}\right|=0\right)$, the transmitter can determine the optimum transmit beamforming vectors through solving the optimization problem below.

$$
\begin{gathered}
{\left[\mathbf{w}_{i}^{*}, \mathbf{w}_{j}^{*}\right]=\arg \max _{\mathbf{w}_{i}:\left\|\mathbf{w}_{i}\right\|=1, \mathbf{w}_{j}:\left\|\mathbf{w}_{j}\right\|=1, \mathbf{w}_{i} \neq \mathbf{w}_{j}}} \\
\log _{2}\left(1+\left|\mathbf{w}_{i}^{H} \mathbf{R}_{i} \mathbf{w}_{i}\right|^{2}\right)+\log _{2}\left(1+\left|\mathbf{w}_{j}^{H} \mathbf{R}_{j} \mathbf{w}_{j}\right|^{2}\right) \\
\text { s.t. }\left|\mathbf{w}_{i}^{H} \mathbf{R}_{i} \mathbf{w}_{j}\right|=0,\left|\mathbf{w}_{j}^{H} \mathbf{R}_{j} \mathbf{w}_{i}\right|=0 .
\end{gathered}
$$

where $\mathbf{R}_{i}, \mathbf{R}_{j}$ are the $N_{t} \times N_{t}$ normalized covariance channel matrices, $\mathbf{w}_{i}^{*}$ and $\mathbf{w}_{j}^{*}$ are the optimum transmit beamforming vectors of size $N_{t} \times 1$. In order to get $\mathbf{w}_{i}^{*}, \mathbf{w}_{j}^{*}$, we apply the theorem proved in [9].

Theorem: $\mathbf{R}_{i} \mathbf{x}_{m}=\alpha_{m} \mathbf{R}_{j} \mathbf{x}_{m}, \mathbf{R}_{j} \mathbf{y}_{n}=\beta_{n} \mathbf{R}_{i} \mathbf{y}_{n}, m, n=$ $1,2, \cdots, N_{t}$. Both $\mathbf{R}_{i}$ and $\mathbf{R}_{j}$ are $N_{t} \times N_{t}$ Hermitan matrices and $\alpha_{m}, m=1,2, \cdots, N_{t}$ are real generalized eigenvalues sorted in ascending order, so is $\beta_{n} . \mathbf{x}_{m}, \mathbf{y}_{n}$ are the corresponding generalized eigenvectors. Then any $\mathbf{x}_{m}, \mathbf{y}_{n}$ can satisfy the zero inter-user interference constraint in equation (7) only if $m, n=1,2, \cdots, N_{t}, m \neq n$. On the condition of $N_{t}=2$, let $\Omega$ be the set of the qualified generalized eigenvector pairs $\left\{\mathbf{x}_{m}, \mathbf{y}_{n}\right\}, \Omega=\left[\left\{\mathbf{x}_{1}, \mathbf{y}_{2}\right\},\left\{\mathbf{x}_{2}, \mathbf{y}_{1}\right\},\left\{\mathbf{y}_{1}, \mathbf{x}_{2}\right\},\left\{\mathbf{y}_{2}, \mathbf{x}_{1}\right\},\left\{\mathbf{x}_{1}, \mathbf{x}_{2}\right\}\right.$, $\left.\left\{\mathbf{x}_{2}, \mathbf{x}_{1}\right\},\left\{\mathbf{y}_{1}, \mathbf{y}_{2}\right\},\left\{\mathbf{y}_{2}, \mathbf{y}_{1}\right\}\right]$. That is, the optimum transmit beamforming vector pair $\left\{\mathbf{w}_{i}^{*}, \mathbf{w}_{j}^{*}\right\}$ is chosen from $\Omega$.

Substituting $\mathbf{w}_{i}, \mathbf{w}_{j}$ in $G_{i, i}$ and $G_{j, j}$ with $\mathbf{w}_{i}^{*}, \mathbf{w}_{j}^{*}$ respectively, we get $G_{i}^{*}$ and $G_{j}^{*}$ which have the similar meanings as that in section IV.

From equation (3), after nulling the inter-user interference $\left(\left|G_{i, j}\right|=0,\left|G_{j, i}\right|=0\right)$, the instant sum-rate during one time slot can be written as

$$
R=\log _{2}\left(1+S I N R_{i}\right)+\log _{2}\left(1+S I N R_{j}\right)
$$

where $S I N R_{i}=\frac{P_{B S}\left|G_{i}^{*}\right|^{2}}{N_{t} d_{i}^{n} \sigma^{2}}, S I N R_{j}=\frac{P_{B S}\left|G_{j}^{*}\right|^{2}}{N_{t} d_{j}^{n} \sigma^{2}}$

\section{B. Design of Frame Structure}

The frames of scheme 1 and scheme 2 contain equally $T$ time slots for comparing their performances.

1) Frame Structure of Scheme 1: The frame structure is shown in Fig 2. Considering PBS and RS serve $K_{A}+K_{B}+K_{C}$ users, our proposed user scheduling strategy enables a near fair utilizing of sum-rate through allocating different proportional time slots to the high and low-rate region. We divide the time slots of the frame into 3 types, named A, B and C. $T_{A_{1}}, T_{B_{1}}$, $T_{C_{1}}$ denotes the number of $\mathrm{A}, \mathrm{B}$ and $\mathrm{C}$ time slots respectively. $T_{A_{1}}, T_{B_{1}}$ and $T_{C_{1}}$ satisfy $T_{A_{1}}+T_{R}=T, T_{R}=2 T_{B_{1}}=2 T_{C_{1}}$. One B time slot contains two different links in the mean time: $\mathrm{PBS} \rightarrow P U_{N}, \mathrm{PBS} \rightarrow \mathrm{RS}$ while $\mathrm{C}$ time slot contains $\mathrm{RS} \rightarrow P U_{F}$, $\mathrm{RS} \rightarrow \mathrm{CU}$

2) Frame Structure of Scheme 2: The frame structure of scheme 2 is the same as that of scheme 1 except that there are no $\mathrm{C}$ time slots. $T_{A_{2}}$ and $T_{B_{2}}$ satisfy that $T_{A_{2}}+T_{B_{2}}=T$, $T_{A_{1}}=T_{A_{2}}, T_{B_{2}}=2 T_{B_{1}}$. In scheme $2, A$ time slot contains two $\mathrm{PBS} \rightarrow P U_{N}$ links whereas $\mathrm{B}$ time slot contains two $\mathrm{PBS} \rightarrow P U_{F}$ links. 


\section{Performance Analysis}

In this section, the user scheduling strategy of the scheme 1 and 2 are illustrated in detail. Considering perfect channel covariance matrix feedback is hard to implement in reality, we then go on to examine the behavior of scheme 1 in the presence of imperfect feedback. Finally, the expressions of sum-rate of scheme 1 with both perfect and imperfect covariance matrix feedback are presented.

\section{A. Sum-rate of Scheme 1 with Perfect $\boldsymbol{R}$ feedback}

We assume that both the PUs and CUs have equipped with GPS, so PBS and RS know the distance of each users, also that the discussion is based on the configuration $N_{t}=N_{r}=2$. Referring to the frame structure, the user scheduling strategy of system 1 can be divided into 3 phases:

1) Sum-rate in each A Time Slot: In each A time slot, PBS selects two PUs whose distances to PBS are shorter than the others in A area and remains other PUs silent in this slot. This method is to achieve as much high sum-rate as possible in that large scale path loss plays a key role in the value of SINR received by the user. Assume the indices of the two selected PUs is $a$ and $b, a, b \in\left[1,2, \cdots, K_{A}\right]$. After using beamforming in PBS, the instant sum-rate in one A time slot is

$$
{ }_{1}^{A} R(t)=\log _{2}\left(1+{ }_{1}^{A} S I N R_{P, a}\right)+\log _{2}\left(1+{ }_{1}^{A} S I N R_{P, b}\right)
$$

where ${ }_{1}^{A} S I N R_{P, a}=\frac{P_{B S}\left|G_{a}^{*}\right|^{2}}{N_{t} d_{a}^{n} \sigma^{2}},{ }_{1}^{A} S I N R_{P, b}=\frac{P_{B S}\left|G_{b}^{*}\right|^{2}}{N_{t} d_{b}^{n} \sigma^{2}}$

2) Sum-rate in each B Time Slot: In each B time slot, one of the antennas in PBS is used to transmit the data to RS to assist the transmission of the PUs in B area, the other is used to serve a PU in A area. PBS select RS(seen as a special PU) and the PU whose distance to PBS is the shortest among all the users in A area, named PU e. $\widetilde{\mathbf{H}_{\Delta}}=d_{\Delta}^{-n} \mathbf{H}_{\Delta}$ denotes the Ricean fading channel model between PBS and RS. The (n,m)-th entry of $\mathbf{H}_{\Delta}$ is

$$
h_{\Delta}(n, m)=\sqrt{\frac{\rho}{\rho+1}} \sigma \exp (j \theta)+\sqrt{\frac{1}{\rho+1}} C N\left(0, \sigma^{2}\right)
$$

where $\theta$ is uniformly distributed in $[0,2 \pi]$. RS and PU $e$ send back their $\mathbf{R}$ to the transmitter. Similarly, the instant sum-rate in one B time slot is

$$
{ }_{2}^{A} R(t)=\log _{2}\left(1+\frac{P_{B S}\left|G_{e}^{*}\right|^{2}}{N_{t} d_{e}^{n} \sigma^{2}}\right)
$$

3) Sum-rate in each C Time Slot: The $\mathrm{C}$ time slots are made to serve the PUs in B area and CUs in $\mathrm{C}$ area while keeping PBS and all PUs in A area silent. Like in the A and B time slots, RS selects one CU in C area whose distance to RS is the shortest among the $K_{C}$ CUs. We name the selected PU and $\mathrm{CU}$ as $q$ and $c$. After using beamforming in the transmitter, the equivalent baseband signal received by RS and the PU $q$ in $\mathrm{B}$ area during $\mathrm{B}$ and $\mathrm{C}$ time slots can be written as $r_{\Delta}, r_{q}$

$$
\begin{aligned}
& r_{\Delta}=G_{\Delta}^{*} \sqrt{\frac{P_{B S}}{d_{\Delta} N_{t}}} s_{p}+\widetilde{n_{\Delta}}, \quad r_{q}=G_{q}^{*} \sqrt{\frac{P_{R S}}{d_{q} N_{t}}} \frac{r_{\Delta}}{\sqrt{E}}+\widetilde{n_{q}} \\
& E\left(\left|r_{\Delta}\right|^{2}\right)=E\left(r_{\Delta}^{H} r_{\Delta}\right)=\left|G_{\Delta}^{*}\right|^{2} \frac{P_{B S}}{d_{\Delta}^{n} N_{t}}+\sigma^{2}=E
\end{aligned}
$$

where $E$ denotes the contribution of the received signal and the noise power. Then $r_{q}$ can be rewritten as

$r_{q}=\sqrt{\frac{P_{R S}}{d_{q}^{n} N_{t}}} \sqrt{\frac{P_{B S}}{d_{\Delta}{ }^{n} N_{t}}} \frac{G_{q}^{*} G_{\Delta}^{*}}{\sqrt{E}} s_{p}+\sqrt{\frac{P_{R S}}{d_{q}^{n} N_{t}}} \frac{G_{q}^{*}}{\sqrt{E}} \widetilde{n_{\Delta}}+\widetilde{n_{q}}$

From the equation (13), the signal power can be written as $E_{S_{q}}=\frac{P_{R S} P_{B S}}{\left(d_{q} d_{\Delta}\right)^{n} N_{t}^{2}} \frac{\left|G_{q}^{*}\right|^{2}\left|G_{\Delta}^{*}\right|^{2}}{E}$, and the noise power states as $E_{N_{q}}=\left(\frac{P_{R S}}{d_{q}^{n} N_{t}} \frac{\left|G_{q}^{*}\right|^{2}}{E}+1\right) \sigma^{2}$, thus we obtain the SINR of the PU q, i.e., ${ }_{3}^{B} S I N R_{P, q}=\frac{E_{S_{q}}}{E_{N_{q}}}$. Likewise, the SINR of CU $c$ is ${ }_{3}^{C} S I N R_{S, c}=\frac{P_{R S}\left|G_{c}^{*}\right|^{2}}{d_{c}^{n} \sigma^{2}}$. The instant sum-rate in one C time slot can be written as

$$
{ }_{3}^{B C} R(t)={ }_{3}^{B} R(t)+{ }_{3}^{C} R(t)
$$

where ${ }_{3}^{B} R(t)=\log _{2}\left(1+{ }_{3}^{B} S I N R_{P, q}\right), \quad{ }_{3}^{C} R(t)=\log _{2}\left(1+{ }_{3}^{C}\right.$ $\left.S I N R_{P, c}\right)$ denotes the instant sum-rate of PUs in B area and CUs in $\mathrm{C}$ area during one $\mathrm{C}$ time slot respectively.

4) Sum-rate of the Entire System of Scheme 1: When N(the number of the frame during simulating) is large, the sumrate of PUs, CUs and the whole system containing both PUs and CUs(denoted as $R_{P}, R_{C}, R_{\text {Total }}^{1}$ ) can be approximately written as

$$
\begin{aligned}
& R_{P}=\frac{1}{N} \sum_{t=1}^{N} \frac{1}{T}\left\{\sum_{T_{A_{1}}}{ }_{1}^{A} R(t)+\sum_{T_{B_{1}}}^{A} R(t)+\sum_{T_{C_{1}}}{ }_{3}^{B} R(t)\right\} \\
& R_{C}=\frac{1}{N} \sum_{t=1}^{N} \frac{1}{T} \sum_{T_{C_{1}}}{ }_{3}^{C} R(t), \quad R_{\text {Total }}^{1}=R_{P}+R_{C}
\end{aligned}
$$

\section{B. Sum-rate of Scheme 2 with perfect $\boldsymbol{R}$ feedback}

Considering there are no $\mathrm{CR}$ network and RS, $T_{B_{2}}$ time slots are used to serve the $P U_{F}$ in $\mathrm{B}$ area. In each time slot of $T_{A_{2}}$ time, PBS selects two PUs in A area whose distances to PBS are shorter than any other PU; in each time slot of $T_{B_{2}}$ time, PBS selects two nearest PUs in B area while keeping all $P U_{N}$ silent. Let ${ }_{N}^{A} R$ and ${ }_{F}^{B} R$ denote the instant sum-rates of each time slot in A and B area, then the sum-rate of the whole system of scheme 2 (denoted as $R_{\text {Totall }}^{2}$ ) can be derived as

$$
R_{\text {Total }}^{2}=\frac{1}{N} \sum_{t=1}^{N} \frac{1}{T}\left\{\sum_{T_{A_{2}}}{ }_{N}^{A} R(t)+\sum_{T_{B_{2}}}^{B}{ }_{F}^{B} R(t)\right\}
$$

\section{Sum-rate in scheme 1 with Imperfect $\boldsymbol{R}$ feedback}

With accurate covariance matrix $\mathbf{R}$ being processed by the transmitter, inter-user interferences can be nulled according to equation (6). However, perfect feedback is not possible in real situations, especially in the system with the coexistence of the primary network and the CR network that share the same spectrum. one reason may be that the CR network is hard to be exactly coordinated with the primary network. So the $\mathbf{R}$ sent back by PUs or CUs may be interfered with each other; the other may be ascribed to the quantization error of the covariance matrix. Now Consider the scheme 1, from the 
discussion above, only in $\mathrm{C}$ time slots can the $\mathrm{CR}$ network be activated to access the licensed spectrum, so to simplify the analysis, we assume that perfect $\mathbf{R}$ feedback will take place in both $\mathrm{A}$ and $\mathrm{B}$ time slots whereas in $\mathrm{C}$ time slots imperfect covariance matrix $\widetilde{\mathbf{R}}$ will be sent back by the selected PU in $\mathrm{B}$ area and the selected $\mathrm{CU}$ in $\mathrm{C}$ area. The expressions of sum-rate in A and B time slots are the same as equation (9) and (11). $\widetilde{\mathbf{R}}$ can be modelled as $\widetilde{\mathbf{R}}=\Delta \mathbf{R} /\|\Delta \mathbf{R}\|_{F}$ where $\Delta \mathbf{R}=\mathbf{R}+\alpha \frac{\|\mathbf{R}\|_{F}}{N_{t}} \Theta, \Theta$ is a $N_{t} \times N_{t}$ matrix with independent complex Gaussian random variables of zero mean and unit variance $C N(0,1)$ and $\alpha$ is a perturbation error factor.

Due to the imperfect covariance matrix feedback, the interuser interference should be taken into account in the calculation of $S I N R$. Let the indices of the selected PU and CU in each $\mathrm{C}$ time slot be $q$ and $c$ respectively, $\Omega$ be the set of the qualified generalized eigenvector pairs of $\widetilde{\mathbf{R}_{q}}$ and $\widetilde{\mathbf{R}_{c}}$ defined in the theorem in section III. The equation (6) is modified as

$$
\begin{aligned}
& {\left[\widetilde{\mathbf{w}_{q}^{*}}, \widetilde{\mathbf{w}_{c}^{*}}\right]=\arg \underset{\mathbf{w}_{q}, \mathbf{w}_{c} \in \Omega, \mathbf{w}_{q} \neq \mathbf{w}_{c}}{ }} \\
& \log _{2}\left(1+\left|\mathbf{w}_{q}^{H} \widetilde{\mathbf{R}_{q} \mathbf{w}_{q}}\right|^{2}\right)+\log _{2}\left(1+\left|\mathbf{w}_{c}^{H} \widetilde{\mathbf{R}_{c} \mathbf{w}_{c}}\right|^{2}\right)
\end{aligned}
$$

After replacing $\mathbf{w}_{q}, \mathbf{w}_{c}$ in $G_{q, q}, G_{q, c}, G_{c, q}$ and $G_{c, c}$ with $\widetilde{\mathbf{w}_{q}^{*}}, \widetilde{\mathbf{w}_{c}^{*}}$ respectively, we get $\widetilde{G_{q}^{*}}, G_{q, c}^{*}, \widetilde{G_{c, q}^{*}}, \widetilde{G_{c}^{*}}$. In each C time slot, the signal received by the PU $q$ and the $\mathrm{CU} c$ after beamforming in the transmitter can be written as

$$
\begin{aligned}
& \widetilde{r_{q}}=\sqrt{\frac{P_{R S}}{d_{q}^{n} N_{t}}} \sqrt{\frac{P_{B S}}{d_{\Delta} N_{t}}} \frac{\widetilde{G_{q}^{*}} G_{\Delta}^{*}}{\sqrt{E}} s_{p}+I_{q, c}+N_{q} \\
& I_{q, c}=\sqrt{\frac{P_{R S}}{d_{q}{ }^{n} N_{t}}} \widetilde{G_{q, c}^{*}} s_{c}, N_{q}=\sqrt{\frac{P_{R S}}{d_{q}^{n} N_{t}}} \frac{\widetilde{G_{q}^{*}}}{\sqrt{E}} \widetilde{n_{\Delta}}+\widetilde{n_{q}} \\
& \widetilde{r_{c}}=\sqrt{\frac{P_{R S}}{d_{c}^{n} N_{t}}} \widetilde{G_{c}^{*}} s_{c}+I_{c, q}+N_{c} \\
& I_{c, q}=\sqrt{\frac{P_{R S}}{d_{c}^{n} N_{t}}} \sqrt{\frac{P_{B S}}{d_{\Delta} N_{t}}} \frac{\widetilde{G_{c, q}^{*}} G_{\Delta}^{*}}{\sqrt{E}} s_{p} \\
& N_{c}=\sqrt{\frac{P_{R S}}{d_{c} N_{t}}} \frac{G_{c, q}^{*}}{\sqrt{E}} \widetilde{n_{\Delta}}+\widetilde{n_{c}}
\end{aligned}
$$

where $I_{q, c}$ is the interference generated by $\mathrm{CU} c$ at the receiver of $\mathrm{PU} q$, other symbols denote the similar meanings in equation (12) and (13). The instant sum-rate with imperfect $\mathbf{R}$ feedback in one $\mathrm{C}$ time slot is

$$
\widetilde{{ }_{3} C} R=\log _{2}\left(1+\frac{E_{S_{q}}}{E_{I_{q, c}}+E_{N_{q}}}\right)+\log _{2}\left(1+\frac{E_{S_{c}}}{E_{I_{c, q}}+E_{N_{c}}}\right)
$$

where $E_{S}, E_{I}$ and $E_{N}$ stands for the the power of signal, interference and noise respectively. Finally, the sum-rate of the whole system of scheme 1 with imperfect feedback is presented as

$\widetilde{R_{\text {Total }}^{1}}=\frac{1}{N} \sum_{t=1}^{N} \frac{1}{T}\left\{\sum_{T_{A_{1}}}{ }_{1}^{A} R(t)+\sum_{T_{B_{1}}}{ }_{2}^{A} R(t)+\sum_{T_{C_{1}}} \widetilde{{ }_{3}^{C}} R(t)\right\}$

From the discussion above, our proposed system of scheme 1 can be seen, to some extent, as a system with the combination of the underlay and overlay CR network: for one thing, by using beamforming approach, the interference generated by the CUs at the PUs is below some acceptable threshold; for another, in spite of the fact that PUs utilize A, B and

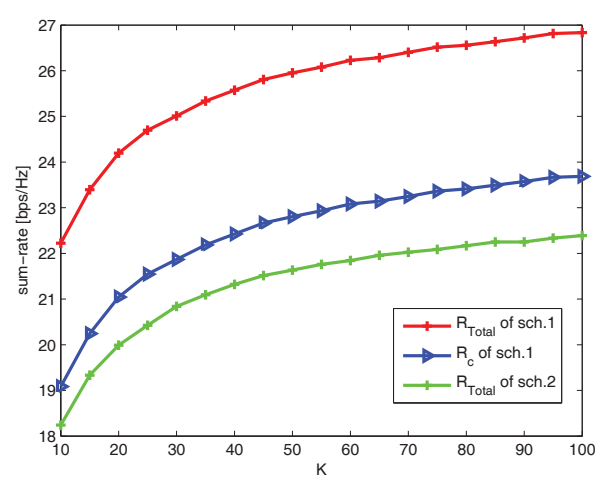

Fig. 3. Scheme 1 vs. Scheme 2

C time slots in the scheduling process, CBS(RS) can assist the transmission of the PUs in the low-rate region in $\mathrm{C}$ time slots while permitting the CR network to start communication simultaneously.

\section{SIMULATION RESULTS}

In this section, we compare the performance of scheme 1 with that of scheme 2 in terms of the sum-rate. The configurations of scheme 1 in our simulation are presented as follows. Both PBS, RS and the PUs and CUs are equipped with two antennas, that is $N_{t}=N_{r}=2$. The transmitting power of PBS and RS $P_{B S}=P_{R S}=10 \mathrm{dBm}$ and the noise power $\sigma_{k}^{2}=-106 \mathrm{dBm}$. We assume the noise variance received by $\mathrm{RS}$, the PUs and the CUs are the same. The radius of A area ranges from $50 m \sim 300 m$ and the radius of $\mathrm{B}$ area ranges from $500 \mathrm{~m} \sim 600 \mathrm{~m}$. The radius of $\mathrm{C}$ area(RS lies in the center of area C) ranges from $50 \mathrm{~m} \sim 200 \mathrm{~m}$. The path loss exponent $n=4$ and the distance between PBS and RS $d_{\Delta}=250 \mathrm{~m}$. Each frame consists $T=10$ time slots. Let prop $_{1}=T_{A_{1}} / T$ and prop $_{2}=T_{A_{2}} / T$ stand for the proportion of time slots allocation between $\mathrm{A}$ and $\mathrm{B}$ area of scheme 1 and scheme 2 respectively. We generate $\mathrm{N}=2000$ frames to calculate the sum-rate. The simulations of Fig.3-Fig.5 are based on the assumption of perfect covariance matrix feedback.

In Fig. 3, the sum-rate of PUs and CUs under the proposed system as a function of the $K$ (we let $K_{A}=K_{B}=K_{C}=\mathrm{K}$ ), for prop $_{1}=$ prop $_{2}=0.4$ is depicted. The sum-rate increases with the total number of users grows which exploits the multiuser diversity. For a large number of users, the chance to have the best users selected gets higher to yield the increase of the sumrate. It can be observed in Fig. 3 that compared with scheme 2 , scheme 1 yields good performance.

Fig.4 presents the change of the sum-rate of scheme 1 caused by the different proportions of time slots allocation for prop $_{1}$ during each frame. We go on the similar simulation as Fig. 3 with prop $_{1}=0.6$ and prop $_{1}=0.4$ respectively. It can be concluded that when the time $\left(T_{A}\right.$ time slots) served for PUs in the high-rate region increases, the sum-rate of the entire system ascends, and that the sum-rate of the cognitive network 


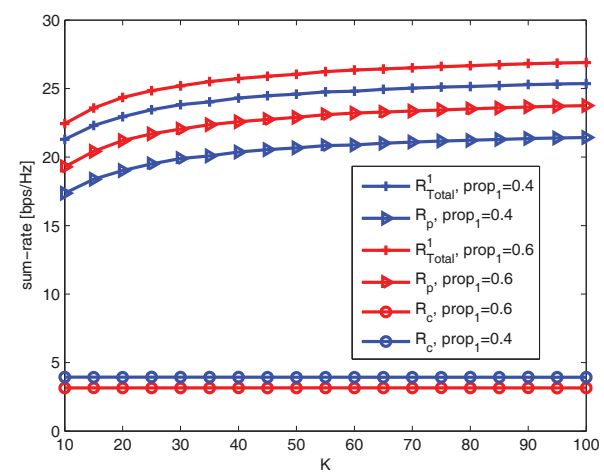

Fig. 4. Effect of distinct allocation of time slots

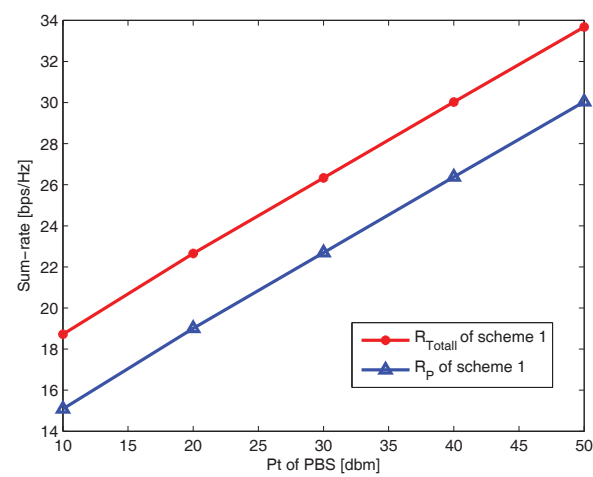

Fig. 5. Sum-rates with different transmitting power

declines a little resulting from the decrease of the number of time slots $\left(T_{C_{1}}\right)$ served for cognitive area.

Fig.5 shows $R_{P}$ and $R_{\text {total }}$ of scheme 1 versus $P_{B S}$ for $K=100$, $_{\text {prop }}=0.4$. It can be seen from the figure that when $P_{B S}$ becomes larger, the total system sum-rate increases. From equation (6) and (7), the sum rate must grows linearly as $P_{B S}$ increases in $\mathrm{dBm}$ due to the zero inter-user interference. The simulation results are definitely consistent with our inferences.

Fig.6 depicted $\widetilde{R_{\text {total }}^{1}}$ of scheme 1 with imperfect $\mathbf{R}$ feedback versus $\alpha$ and $K$ for prop $_{1}=0.4$ and $P_{B S}=10 \mathrm{dBm}$. It can be seen that when $\alpha=0.1$, the total sum-rate is about 3 $\mathrm{bps} / \mathrm{Hz}$ less than that of $\alpha=0$ (perfect feedback) which states that our system of scheme 1 has certain tolerance to imperfect feedback.

\section{CONCLUSION}

In this work, we consider the three-phase joint scheduling and transmitting scheme in cognitive radio networks. A fixed relay station, serving as a cognitive basestation during the third phase, is deployed in order to improve the throughput of the primary users in the low-rate region while supporting cognitive users' communication with the overlay cognitive pattern. Specially, both primary users in the low-rate region

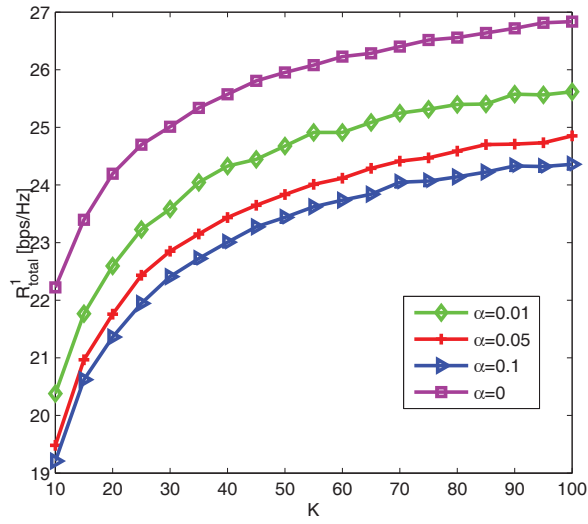

Fig. 6. total sum-rate of the scheme 1 with imperfect feedback

and cognitive users in cognitive area are scheduled to increase sum-rate of the entire system. Furthermore, in order to reduce the feedback and exploit beamforming at transmitting side, all selected users return their covariance matrices to primary base station and/or relay station. As is seen from the simulation results, the proposed scheme (scheme 1) is capable of enhancing the throughput over the basic scheme 2.

\section{ACKNOWLEDGMENT}

This work was supported by the open research fund of $\mathrm{Na}-$ tional Mobile Communications Research Laboratory, Southeast University and the Jiangsu Postdoctoral Research Programs (0801054B) and partially by National Natural Science Foundation of China (No. 60872104) and the Key Project of Natural Science Research Program of Universities, Jiangsu Province (08KJD510001).

\section{REFERENCES}

[1] Federal Communication Commission," Spectrum Policy Task Force," Rep.ET Docket no.02-135,Nov.2002.

[2] J.Mitola and G.Q.Maguire,"Cognitive radio:Making software radios more personal,'IEEE Pers.Commun.,vol.6,pp.13-18,Aug.1999.

[3] A.Goldsmith,S.A.Jafar,I.Maric and S.Srinivasa,'Breaking spectrum gridlock with cognitive radios:an information theoretic perspective,'IEEE Proc.,vol.97,pp.894-914,May.2009.

[4] S.Kim,W.Choi,Y.Choi,J.Lee,Y.Han and I.Lee,"Downlink performance analysis of cognitive radio based cellular relay networks,"IEEE CrownCom2008,pp.1-6,May.2008.

[5] E.Costa,A.Frediani,S.Redana and Y.Zhang,"Dynamic spatial resource sharing in relay enhanced cells,"European Wireless Conference 2007.

[6] T.Yoo and A.Goldsmith,"On the optimality fo multi-antenna broadcast scheduling using zero-forcing beamforming," IEEE J.Select.Areas Commun.,vol.24,pp.528-541,Mar.2006.

[7] C.B.Peel,B.M.Hochwald and A.L.Swindlehurst,"A vector-perturbation technique for near capacity multiantenna multiuser communication-part I:channel inversion and regularization,'IEEE Trans.Comm., vol.53,pp.195202,Jan.2005.

[8] K.Hamdi,W.Zhang and K.B.Letaief,'Low-complexity antenna selection and user Scheduling in cognitive MIMO broadcast systems,"in Proc.IEEE ICC,pp.4038-4042,2008.

[9] C.Chae,D.Mazzarese,N.Jindal,and R.W.Heath,Jr,"A low complexity linear multiuser MIMO beamforming system with limited feedback," IEEE CISS 2008,pp.418-422,Mar.2008. 